\title{
Evidence for the Ventral Origin of Oligodendrocyte Precursors in the Rat Spinal Cord
}

\author{
Benjamin C. Wart, Juin Fok-Seang, and Robert H. Miller \\ Department of Neurosciences, Case Western Reserve University School of Medicine, Cleveland, Ohio 44106
}

\begin{abstract}
The neuroepithelial cells of the mammalian neural tube are thought to give rise to all classes of differentiated neurons and macroglial cells in the adult CNS. In most cases, the regulation and timing of commitment of neuroepithelial cells to specific differentiative pathways are unknown. It has been proposed that in developing spinal cord, the macroglial cells-astrocytes and oligodendrocytes-arise either by the direct transformation of radial glial cells in the developing cord or, alternatively, by the differentiation of distinct precursor cells which migrate to presumptive white matter from the region of the central canal during development. In this study, the timing of oligodendrocyte differentiation in different levels of the spinal cord and the capacity of specific regions of the spinal cord to give rise to oligodendrocytes at various ages was tested in vitro. At embryonic day 14, all complete segments, as well as all ventral regions along the rostral-caudal axis of the spinal cord, have the capacity for oligodendrogenesis. By contrast, dorsal regions of the thoracic and lumbar spinal cord do not develop the capacity for oligodendrogenesis until later in development. The capacity of dorsal rat spinal cord to give rise to oligodendrocytes appears to be associated with the ventral-to-dorsal migration of oligodendrocyte precursors. These observations suggest that commitment to an oligodendrocyte differentiative pathway appears to occur in a distinct population of ventrally located glial precursors in the embryonic rat spinal cord.
\end{abstract}

The spinal cord has provided a useful model for the study of CNS development since the early work of Retzius (1898) and Ramon y Cajal (1909). Although the development (Nornes and Das, 1974; Altman and Bayer, 1984) and organization (Rexed, 1952; Brown, 1981) of neurons in the vertebrate spinal cord has been extensively studied and is comparatively well understood, the origin of spinal cord macroglial cells is less clear.

Like other regions of the CNS, the spiral cord contains two major classes of macroglial cells, oligodendrocytes and astrocytes. While oligodendrocytes are the myelinating cell of the CNS (Bungc, 1968; Peters et al., 1976), the functions of astrocytes are not well defined. However, astrocytes have been proposed to play a role in supporting axonal outgrowth (Silver et al., 1982; Smith et al., 1990) and neuronal cell migration (Rakic, 1971; Hatten, 1990), formation of the blood brain barrier (Jan-

\footnotetext{
Received Dec. 17, 1990; revised Mar. 12, 1991; accepted Mar. 13, 1991

We thank Drs. A. Hall and S. Landis for helpful comments on the manuscript. This work was supported by NIH Grant NS-25597-02 and the American Multiple Sclerosis Society. R.H.M. is an Alfred P. Sloan fellow.

Correspondence should be addressed to Dr. Miller at the above address.

Copyright (c) 1991 Society for Neuroscience 0270-6474/91/112477-12\$03.00/0
}

zer and Raff, 1987), and maintenance of the extracellular ionic environment (Hertz, 1981).

Previous studies have examined the proliferation and differentiation of spinal cord astrocytes and oligodendrocytes during late embryonic and early postnatal stages of development (Fujita, 1965; Gilmore, 1971; Ling, 1976; Sturrock, 1982). Consistent with the developmental sequence in other regions of the CNS, the majority of neurons in developing spinal cord undergo their final division before the majority of glial cells (Altman and Bayer, 1984). Furthermore, the majority of astrocytes differentiate before the majority of oligodendrocytes (Ling, 1976), and both astrocytes and oligodendrocytes or their precursors continue to proliferate postnatally (Gilmore, 1971).

Morphological and immunocytochemical analyses of the developing spinal cord have resulted in two distinct hypotheses to explain the origin of astrocytes and oligodendrocytes. First, it has been proposed that, as in forebrain (Smart, 1961; Privat and Leblond, 1972), immature glial cells develop first in the region of the central canal and subsequently migrate to the white matter, where they proliferate and differentiate into mature astrocytes or oligodendrocytes (Fujita, 1965; Gilmore, 1971). Alternatively, it has been proposed that both astrocytes and oligodendrocytes differentiate directly from the embryonic radial glial cells of the developing spinal cord (Choi et al., 1983; Choi and Kim, 1985; Hirano and Goldman, 1988). While there is good evidence that in other parts of the CNS radial glial cells share a number of properties with astrocytes (Schmechel and Rakic, 1979; Levitt and Rakic, 1980) and may be the direct precursor of at least some astrocytes (Culican et al., 1990), it is less clear that radial glial cells represent the immediate precursor for oligodendrocytes. Recently, radially oriented cells in the presumptive white matter of postnatal rat spinal cords were shown to express antigens characteristic of oligodendrocytes as well as of radial glia. These observations provide some support for the radial glial origin of oligodendrocytes (Hirano and Goldman, 1988).

Tissue culture techniques have helped to elucidate certain aspects of CNS glial cell development (Culican et al., 1990; Raff, 1989). For example, in cultures of neonatal rat optic nerve, three distinct types of differentiated macroglial cells have been identified (Raff, 1989): oligodendrocytes, which have a large number of branched processes and are the only cells which react with antibodies against galactocerebroside (GC) (Raff et al., 1978; Ranscht et al., 1982); and two distinct types of astrocytes, called type-1 and type-2 astrocytes, both of which contain glial fibrillary acidic protein (GFAP + ) intermediate filaments (Bignami et al., 1972; Bignami and Dahl, 1974; Pruss, 1979).

Most type-1 astrocytes have few processes, are Ran-2 (Bartlett et al., 1981) immunoreactive but lack A2B5 (Eisen- 
barth et al., 1979) reactivity. By contrast, most type-2 astrocytes have a process-bearing morphology and label with A2B5 but not with Ran-2 (Raff et al., 1983a). These three macroglial cells develop from two separate lineages (Raff et al., 1984). One lineage gives rise to type-1 astrocytes, while in the second lineage a bipotential (0-2A) progenitor cell develops into either oligodendrocytes or type-2 astrocytes depending on the culture environment (Raff et al., 1983b; Hughes and Raff, 1987; Lillien and Raff, 1990). Two types of astrocyte with characteristics similar to type- 1 and type- 2 optic nerve astrocytes (Williams et al., 1985; Levi et al., 1986a), as well 0-2A progenitor-like cells (Behar et al., 1988; Levi et al., 1986b), have been found in cultures of rat cerebrum and cerebellum, suggesting that such glial cell heterogeneity is a common feature of the rodent CNS.

The timing of glial cell differentiation does not appcar to depend on CNS morphogenesis, since it can be reconstituted in vitro in the absence of morphogenetic cues. In dissociated cell cultures, type- 1 astrocytes, ependymal cells, oligodendrocytes, and type- 2 astrocytes develop on the same schedule as they do in vivo (Abney et al., 1981; Williams et al., 1985; Lillien and Raff, 1990). These observations have led to the proposal that CNS glial cell differentiation is primarily regulated by cellular biological clocks that operate independently of positional information (Abney et al., 1981; Williams et al., 1985).

We have examined the timing of oligodendrocyte differentiation in the rat spinal cord and assessed the capacity of different regions of spinal cord to give rise to oligodendrocytes in vitro, at various stages of development. We show that oligodendrocyte differentiation follows both a rostral-caudal and ventral-dorsal sequence and that each segment of the spinal cord has the capacity to give rise to oligodendrocytes when cultured at embryonic day 14 (E14). More importantly, in E14 thoracolumbar segments, the capacity to give rise to oligodendrocytes is restricted to ventral regions of the spinal cord. Later in development, dorsal spinal cord regions appear to aquire the capacity for oligodendrogenesis through the ventral-to-dorsal migration of oligodendrocyte precursor cells.

\section{Materials and Methods}

Preparation of cell suspensions. Embryonic Sprague-Dawley rat spinal cords between the ages of E15 and postnatal day 0 (P0; E22) were separated into cervical, thoracic, and lumbar segments, and each segment was further separated into dorsal or ventral regions. Cell suspensions were prepared by mechanical dissociation. The tissue was chopped into small pieces, triturated through a fire-polished Pasteur pipette, passed through a $30-\mu \mathrm{m}$ nylon filter, and plated for $4 \mathrm{hr}$ on poly-Llysine-coated coverslips as previously described (Raff et al., 1984). To identify oligodendrocytes, cells were labeled for $30 \mathrm{~min}$ with a mouse monoclonal antibody against galactocerebroside (anti-GC) [ascites fluid 1:400 in Dulbecco's modified Eagle's medium (DMEM)] (Ranscht et al., 1982), followed by goat anti-mouse Is conjugated to rhodamine (Cappel 1:200). Cells were then fixed in 5\% glacial acetic acid in methanol at $-20^{\circ} \mathrm{C}$, rinsed, and examined with epifluorescence illumination un a Nikon Optiphot microscope. Cell suspensions from at least threc different litters, composed of not less than $1.5 \times 10^{6}$ cells at each age, were analyzed, and the first appearance of GC+ cells was recorded for each different region of the spinal cord.

Preparation of dorsal and ventral spinal cord cultures. Dorsal or ventral regions of thoracolumbar spinal cord from E14, E16, or E18 rats were separated along the sulcus limitans, the meninges were removed, and cells were dissociated as previously described (Smith et al., 1990). Briefly, spinal cords were incubated in calcium-magnesium free buffer (MEMCMF) containing $0.025 \%$ trypsin for $30 \mathrm{~min}$ at $37^{\circ} \mathrm{C}$. Cells were dissociated by trituration through fire-polished Pasteur pipette, pelleted at $1000 \times g$ and resuspended in DMEM-F12 with $10 \%$ fetal bovine serum (FBS). Debris and nondissociated cells were removed by passage through a $30-\mu \mathrm{m}$ nylon filter, and the cells plated at a density of either 0.5 or 1 $\times 10^{6}$ viable cells/well on poly-L-lysine coated $12-\mathrm{mm}$ diameter coverslips. After $12 \mathrm{hr}$, cultures were switched to N2 medium (Bottenstein and Sato, 1979) containing 1\% FBS.

To assay cultures for the presence of oligodendrocytes and astrocytes. cells were labeled before fixation with anti-GC followed by goat antimouse Ig conjugated to rhodamine. After fixation and permeabilization with $5 \%$ glacial acetic acid in methanol at $-20^{\circ} \mathrm{C}$ for $10 \mathrm{~min}$, cells were labeled with rabbit anti-GFAP antibody (Accurate 1:200), followed by goat anti-rabbit Ig conjugated to fluorescein (Cappel 1:200). In all cases antibodies were diluted in DMEM containing $10 \%$ normal goat serum (NGS) and incubations were for $30 \mathrm{~min}$ at room temperature.

To determine when oligodendrocytes first appeared in vitro, cultures were labeled each day after plating. For quantitative studies, oligodendrocyte number was determined at the equivalent age of $\mathrm{PO}$, a time when many GC+ cells were found in all regions of the spinal cord. To ensure that oligodendrocyte differentiation was not simply delayed in E14 dorsal spinal cord cultures, some cultures were allowed to develop until the equivalent of P10 before labeling with anti-GC. Cocultures of dorsal and ventral spinal cord cells were assayed using similar procedures. In some experiments, platelet-derived growth factor - AA (PDGFAA) (Collaborative Research) was added to the appropriate cultures at a concentration of $10 \mathrm{ng} / \mathrm{ml}$ (Noble et al., 1988) for the duration of the experiment. Cultures were examined on a Nikon Optiphot microscope equipped with epifluorescence illumination, and images were recorded on Tri-X film at 400 ASA. Deletion of the primary antibodies or substitution of normal mouse or rabbit serum resulted in the complete absence of specific staining.

Preparation of explant cultures. - Thoracolumbar spinal cords were dissected from E14 embryos, the meninges were removed, and cords were separated along the sulcus limitans. Explant cultures were prepared by chopping the tissue into small fragments, passing tissue fragments through $500-\mu \mathrm{m}$ nylon mesh at least twice, and plating the resultant fragments on $12-\mathrm{mm}$ poly-L-lysine-coated coverslips in a small amount of medium at a density of approximately 20 explants/coverslip. After $9 \mathrm{~d}$ in N2 medium $+1 \%$ FBS, cultures were double-labeled with antiGC and anti-GFAP antibodies as described above.

To define the antigenic characteristics of cells migrating from E14 ventral explants, cultures were labeled after $5 \mathrm{~d}$ with anti-GC, antiGFAP, and the monoclonal antibody A2B5, which identifies 0-2A progenitor cells in the optic nerve. A2B5 labeling was performed on cells prior to fixation using ascites fluid diluted 1:100 in DMEM + 10\% NGS for 30 min followed by goat anti-mouse Ig conjugated to rhodamine.

To determine whether coculture of dorsal spinal cord cells with nonCNS, ventrally derived tissue resulted in oligodendrocyte development, explants were also established from E14 notochord or ventral tissue masses (including heart and ventral somites). These explants were then cocultured with $0.5 \times 10^{6} \mathrm{E} 14$ dorsally derived spinal cord cells for 9 $\mathrm{d}$ in N2 medium and then labeled with anti-GC and anti-GFAP as described abovc.

Determination of oligodendrocyte cell number. To determine number of oligodendrocytes that developed in cultures of dissociated spinal cord, cells were labeled with anti-GC and the number of GC+ cells was counted in 10 consecutive fields under a $20 \times$ objective using rhodamine filters. Counts were then adjusted to reflect the area occupied by all cells on the coverslip. In all cases, the results represent the means \pm SD of cell counts from at least two different coverslips taken from each of three separate experiments. In Table 2, cell counts were taken from cultures originally plated at a density of $1 \times 10^{6}$ viable cells/coverslip and allowed to develop until the equivalent age of P1. In Table 2, cell counts were made at E14+9 (an equivalent age of P1) by an individua! not informed of culture conditions.

Analysis of ventral-to-dorsal cell migration. To examine the ventralto-dorsal migration of oligodendrocytc precursor cells, two different approaches were taken. First, spinal cords were removed from E18 rat embryos, the ventral meninges were split, and a small crystal of 1,1 diactadecyl-3,3,3,3-tetramethyl indocarbocyamine perchlorate (DiI) (Honig and Hume, 1986; Godement et al., 1987) was placed on the exposed region and allowed to remain for $10 \mathrm{~min}$ before being removed. The cord was then washed, cut into small segments, and grown in organ culture in DMEM-F12 + 10\% FBS for $18 \mathrm{hr}$ before being fixed by immersion in $4 \%$ paraformaldehyde in $0.1 \mathrm{~m}$ phosphate buffer $(\mathrm{pH} 7.4)$ containing $7 \%$ sucrose for $1 \mathrm{hr}$ at room temperature. Following fixation, spinal cord segments were incubated in $1 \mathrm{~m}$ sucrose in the same buffer for $30 \mathrm{~min}$. Tissue was then frozen, and $30-\mu \mathrm{m}$ transverse sections were collected on gelatin-subbed slides, mounted in Gelvatol (Monsanto), and examined with rhodamine optics. In a second series of experiments, 
Table 1. Initial appearance of GC I oligodendrocytes in cell suspensions of developing rat spinal cord

\begin{tabular}{|c|c|c|c|c|c|}
\hline \multirow[b]{3}{*}{ Age } & \multicolumn{5}{|c|}{ Segmental level and region of spinal cord } \\
\hline & \multirow[t]{2}{*}{ Cervical } & \multicolumn{2}{|c|}{ Thoracic } & \multicolumn{2}{|l|}{ Lumbar } \\
\hline & & Ventral & Dorsal & Ventral & Dorsa \\
\hline E15 & - & - & - & - & - \\
\hline E16 & + & - & - & - & - \\
\hline E17 & + & + & - & - & - \\
\hline E18 & + & + & + & $+1-$ & - \\
\hline E19 & + & + & + & + & $+1-$ \\
\hline PO & + & + & + & + & + \\
\hline
\end{tabular}

Oligodendrocyte maturation followed both a rostral-caudal and ventral-dorsal temporal sequence. GC+ oligodendrocytes were first seen in the cervical level spinal cord at E16, thoracic level at E17, and lumbar level at E18. In both thoracic and lumbar regions, GC + cells appeared approximately $1 \mathrm{~d}$ earlier in ventral than in dorsal regions. Cell suspensions were prepared by mechanical dissociation, plated on poly-L-lysine-coated coverslips and labeled $4 \mathrm{hr}$ later with antiGC antibodies by indirect immunofluorescence. Less than $5 \%$ of spinal cord cells were GC + in any cell suspension. The absence of significant numbers of oligodendrocytes ( $<5 /$ coverslip) is denoted by - and the presence of more than 100 by + . Animals from three litters at each age were examined.

segments of freshly dissected E21 spinal cord were cut by hand, labeled on the ventral surface with a crystal of DiI for $10 \mathrm{~min}$, and thoroughly washed. The segments were then cultured for up to $18 \mathrm{hr}$, washed, and labeled with anti-GC antibody followed by goat anti-mouse Ig conjugated to fluorescein as described above. Following staining, the segments were fixed in $4 \%$ paraformaldehyde, mounted in Gelvatol, and examined by epifluorescence, microscopy. To control for initial nonspecific localization of the DiI label, some segments were analyzed immediately after labcling. To control for diffusion through the tissue, some segments were fixed immediately after labeling and assayed $18 \mathrm{hr}$ later. In both cases, DiI labeling was restricted to the ventral region of the spinal cord.

\section{Results}

\section{Developmental sequence of oligodendrocyte} differentiation in vivo

To determine when differentiated oligodendrocytes first appeared in each level of the developing spinal cord, cell suspensions were prepared from dorsal and ventral regions of isolated cervical, thoracic, or lumbar segments of spinal cord from animals between embryonic day 15 (E15) and postnatal day 2 (P2). The dissociated cells were labeled by indirect immunofluorescence with anti-GC antibodies to identify oligodendrocytes. The results of these experiments are summarized in Table 1. In cell suspensions from E15 spinal cord, no GC+ cells were found at any segmental level. Significant numbers of GC + cells were first seen in cell suspensions from cervical segments at E16, thoracic segments at E17, and lumbar segments at E18. In addition to the rostrocaudal gradient, in thoracic and lumbar segments, $\mathrm{GC}+$ oligodendrocytes were detected approximately $1 \mathrm{~d}$ earlier in cell suspensions of ventral cord than in those from dorsal cord. For example, in cell suspensions of dorsal-thoracic spinal cord, GC + cells were first seen at E18, and in cell suspensions of dorsal-lumbar spinal cord at E19. At birth (P0), many GC+ cells were present in cell suspensions prepared from all regions of the spinal cord. These results demonstrate that oligodendrocyte differentiation follows both a rostrocaudal and ventrodorsal sequence of differentiation similar to that described for the other major cell classes in the spinal cord (Kingsbury, 1926; Nornes and Das, 1974; Sturrock, 1982).

\section{Development of oligodendrocytes in vitro}

Spinal cord oligodendrocyte development in vitro follows a time course similar to that observed in vivo. For example, when cul- tures prepared from complete thoracolumbar spinal cord at either E14 or E16 were allowed to mature in culture, GC+ cells first appeared at the equivalent of E17, that is, E14+3 days in vitro (DIV), or E16+1DIV. By the equivalent of P1, numerous $\mathrm{GC}+$ oligodendrocytes were seen in all cultures. Thus, between E14 and birth, the timing of oligodendrocyte development in the rat spinal cord does not appear to be dependent on positional information present only in the intact spinal cord.

Although oligodendrocytes developed on schedule in cultures derived from E1 4 complete spinal cord segments, the potential for oligodendrogenesis was not present throughout the whole cord but was restricted to ventral regions at this age. When cultures from ventral or dorsal E14 thoracolumbar spinal cord were labeled with anti-GC and anti-GFAP antibodies at the equivalent age of $\mathrm{P} 1,(\mathrm{E} 14+9 \mathrm{DIV})$, a stage when numerous $\mathrm{GC}+$ cells were found in cell suspensions from all regions of the spinal cord, the cellular compositions of the two cultures were different. While cultures from both ventral and dorsal spinal cord cultures werc denscly populated with neural cells including GFAP+ astrocytes, only the ventrally derived cultures contained significant numbers of GC+ oligodendrocytes (Fig. 1). Furthermore, significant numbers of oligodendrocytes failed to develop in E14 dorsally derived spinal cord cultures even when the cultures were allowed to mature to the equivalent of P10 (E14+19DIV).

While oligodendrocytes did not develop in cultures of E14 thoracolumbar dorsal spinal cord, they did develop from dorsally derived cultures established from older animals. For example, by the equivalent of Pl, GC + cells were seen in both ventrally and dorsally derived cultures established from animals at either E16 or E18 (Table 2). Many more oligodendrocytes, however, developed in cultures derived from E1 8 dorsal spinal cord than from E16. Thus, although isolated dorsal thoracolumbar spinal cord lacked the capacity for oligodendrogenesis at E14, it had acquired that capacity by E16.

\section{Development of ventrally derived oligodendrocytes is unaffected by coculture with dorsal cells}

The absence of oligodendrocytes in E14 dorsally derived spinal cord cultures could be due to the presence of a dorsally located inhibitory signal or to the lack of a ventrally derived inductive 

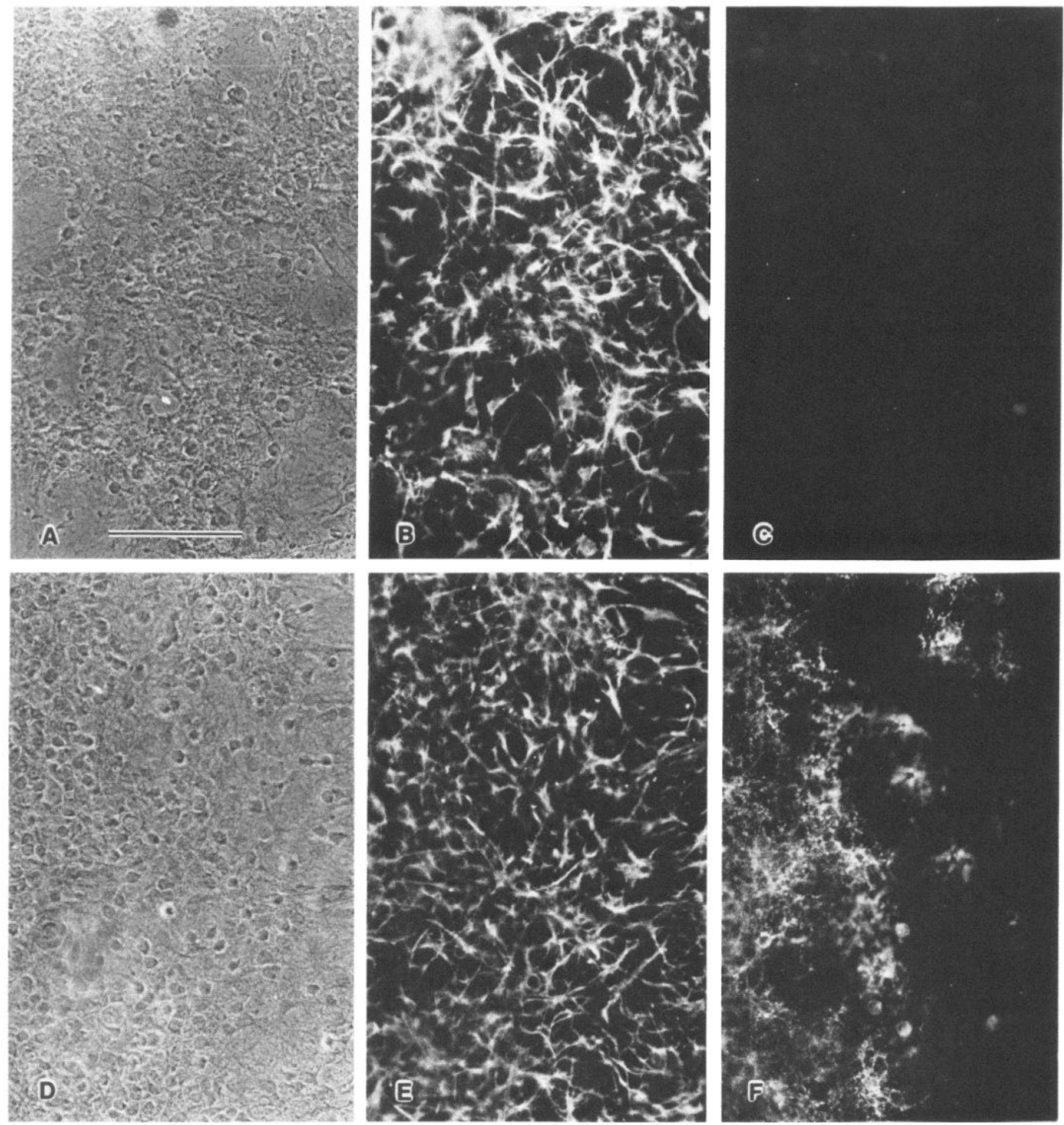

Figure 1. Dissociated cell cultures from E14 dorsal $(A-C)$ or ventral $(D-F)$ rat spinal cord after $9 \mathrm{~d}$ in culture. Cultures are shown with phase optics $(A$ and $D)$ and following double labeling by indirect immunofluorescence with anti-GFAP antibodies to identify astrocytes $(B$ and $E)$ and anti-GC antibodies to identify oligodendrocytes $(C$ and $F)$. While both sets of cultures contain large numbers of neural cells $(A$ and $D)$ including GFAP+ astrocytes $(B$ and $E)$, only the ventrally derived cultures contained GC+ oligodendrocytes $(C$ and $F)$. Scale bar, $100 \mu \mathrm{m}$.

signal. To examine these possibilities, a number of experiments were performed. First, a constant number of E14 ventrally derived spinal cord cells were grown either alone or in the presence of increasing numbers of E14 dorsally derived cells. The number of GC+ oligodendrocytes that developed in each condition was assayed at the equivalent of P1 (E14+9DIV). No significant change in the number of oligodendrocytes was seen following the addition of increasing numbers of E14 dorsally derived cells compared to that seen in cultures containing only ventrally derived cells (Table 3). Second, astrocytes have been shown to produce PDGF (Richardson et al., 1988), which is a potent mitogen for oligodendrocyte precursor cells in other systems 
Table 2. Oligodendrocyte development in dorsal or ventral spinal cord cultures

\begin{tabular}{|c|c|c|}
\hline \multirow{2}{*}{$\begin{array}{l}\text { Initial age }+ \\
\text { days in culture }\end{array}$} & \multicolumn{2}{|c|}{ Number of GC+ cells } \\
\hline & Dorsal & Ventral \\
\hline $\mathrm{E} 14+9$ & $86 \pm 110$ & $17,100 \pm 2800$ \\
\hline $\mathrm{E} 16+7$ & $1570 \pm 30$ & $24,850 \pm 1871$ \\
\hline$E 18+5$ & $12,400 \pm 1650$ & $21,500 \pm 2989$ \\
\hline
\end{tabular}

AT E 14, large numbers of oligodendrocytes developed in ventrally derived cultures but few oligodendrocytes developed in dorsally derived cultures. By E18, significant numbers of oligodendrocytes developed in both ventrally and dorsally derived cultures. Thoracolumbar segments of embryonic rat spinal cords were separated at the sulcus limitans, dissociated into single cells, plated at a density of 1 $\times 10^{6}$ viable cells/coverslip, and allowed to develop until the equivalent age of P1. Cultures were then labeled with anti-GC to identify oligodendrocytes (as described in Materials and Methods), and the total number of oligodendrocytes in each culture was determined. Results indicate the mean \pm SD of counts from two different coverslips taken from each of three separate experiments.

(Noble et al., 1988). Since dorsal spinal cord cells contained many astrocytes that may alter the level of PDGF in these cocultures, ventrally derived spinal cord cells were also grown in the presence of $10 \mathrm{ng} / \mathrm{ml} \mathrm{PDGF}$, and the number of oligodendrocytes was assessed as above. Again, no significant differences were found in the number of oligodendrocytes that subsequently developed. These results suggest that even in the presence of ventrally derived cells, E14 dorsal spinal cord cells did not give rise to oligodendrocytes, and that the number of ventrally derived oligodendrocytes was not substantially altered by coculture with dorsally derived cells. Finally, to determine whether a source of an oligodendrocyte induction signal resided ventrally, but outside the spinal cord, E14 dorsally derived spinal cord cells were cocultured with explants of notochord or other non-CNS tissue from around the ventral spinal cord region of the same-aged animals. After $9 \mathrm{~d}$ in vitro, numerous astrocytes were seen in these cultures, but no oligodendrocytes developed (data not shown). Thus, the lack of oligodendrogenesis in E14 dorsally derived spinal cord cultures does not appear to be due to the absence of a ventrally derived inductive signal or to the presence of a dorsally located inhibitory signal.

\section{Oligodendrocytes do not develop in E14 dorsal spinal cord explant cultures}

An alternative explanation for the lack of oligodendrocytes in E14 dorsally derived spinal cord cultures is that their development is dependent on local cellular interactions that occur later in dorsal than in ventral regions of the developing spinal cord and were disrupted by cell dissociation at E14. To address this possibility, explant cultures, which preserve local cytoarchitecture, were established from dorsal or ventral E14 spinal cord. These explants were allowed to mature in vitro until the equivalent of P1 (E14+9DIV), and the appearance of GC+ oligodendrocytes and GFAP + astrocytes was assayed by indirect immunofluorescence. Large numbers of GFAP + astrocytes developed from both dorsal and ventral explants (Fig. 2). By contrast, while oligodendrocytes developed from the majority of ventral explants $(187 / 332$ or $56 \%)$, less than $1 \%$ of the dorsal explants gave rise to any $\mathrm{GC}+$ cells $(3 / 363)$. In ventral explant cultures, many of the oligodendrocytes that developed did so on the surrounding substrate (Fig. 2), suggesting that immature oligodendrocytes or their precursors migrated out of the main bulk of the explant. It is unlikely that the lack of oligodendrocyte development in E14 dorsally derived spinal cord cultures is due to the disruption of local cell-cell interactions, since the local cytoarchitecture is comparatively well preserved in explant cultures. Not all ventral explants gave rise to oligodendrocytes, suggesting that even in this region of spinal cord oligodendrocyte precursors are not uniformly distributed.

To determine whether the cells that migrated from the explant had the characteristics of immature oligodendrocytes or their precursor cells, E14 ventral explant cultures were labeled after $5 \mathrm{~d}$ in culture with A2B5, anti-GC, and anti-GFAP antibodies. At this stage, the majority of the cells that had migrated from the bulk of the explant had a process-bearing morphology and were immunoreactive with A2B5, but not with anti-GC or GFAP (Fig. 3). This antigenic phenotype is more characteristic of $\mathrm{O}-2 \mathrm{~A}$ progenitor cells than immature oligodendrocytes and is consistent with the migration of precursor cells rather than immature oligodendrocytes.

\section{Ventral-to-dorsal migration of oligodendrocyte precursors}

A likely explanation for the temporal difference in the ability of ventral and dorsal spinal cord to give rise to oligodendrocytes

Table 3. Number of oligodendrocytes that developed from a constant number of E14 ventral spinal cord cells in coculture with dorsal cells.

Number of

$\mathrm{GC}+$

cells at E14+9

Culture conditions (Pl equivalent)

$0.5 \times 10^{6} \mathrm{E} 14$ ventral cells

$0.5 \times 10^{6} \mathrm{E} 14$ ventral cells

$0.5 \times 10^{6} \mathrm{E} 14$ ventral cells

$0.5 \times 10^{6} \mathrm{E} 14$ ventral cells

0 E14 ventral cells

$0.5 \times 10^{6} \mathrm{E} 14$ ventral cells
+0 dorsal cells

$+0.25 \times 10^{6}$ dorsal cells

$+0.5 \times 10^{6}$ dorsal cells

$+0.75 \times 10^{6}$ dorsal cells

$+0.5 \times 10^{6}$ dorsal cells

$+10 \mathrm{ng} / \mathrm{ml}$ PDGF
$12,077 \pm 2380$

$11,573 \pm 2265$

$10,860 \pm 1625$

$10,943 \pm 1082$

$210 \pm 142$

$11,120 \pm 1835$

The number of oligodendrocytes that develop from a constant number of ventrally derived El4 spinal cord cells is not altered by coculture with variable numbers of dorsally derived cells nor by the addition of PDGF. Dissociated cell cultures were prepared from dorsal or ventral spinal cord, and the total number of GC+ oligodendrocytes that developed in each culture was determined as described in Materials and Methods. The results represent means and standard deviations of cell counts from at least two different coverslips taken from each of three separate experiments. Cell counts were made at $\mathrm{E} 14+9$ (an equivalent age of $\mathrm{Pl}$ ) by an individual not informed of culture composition. 

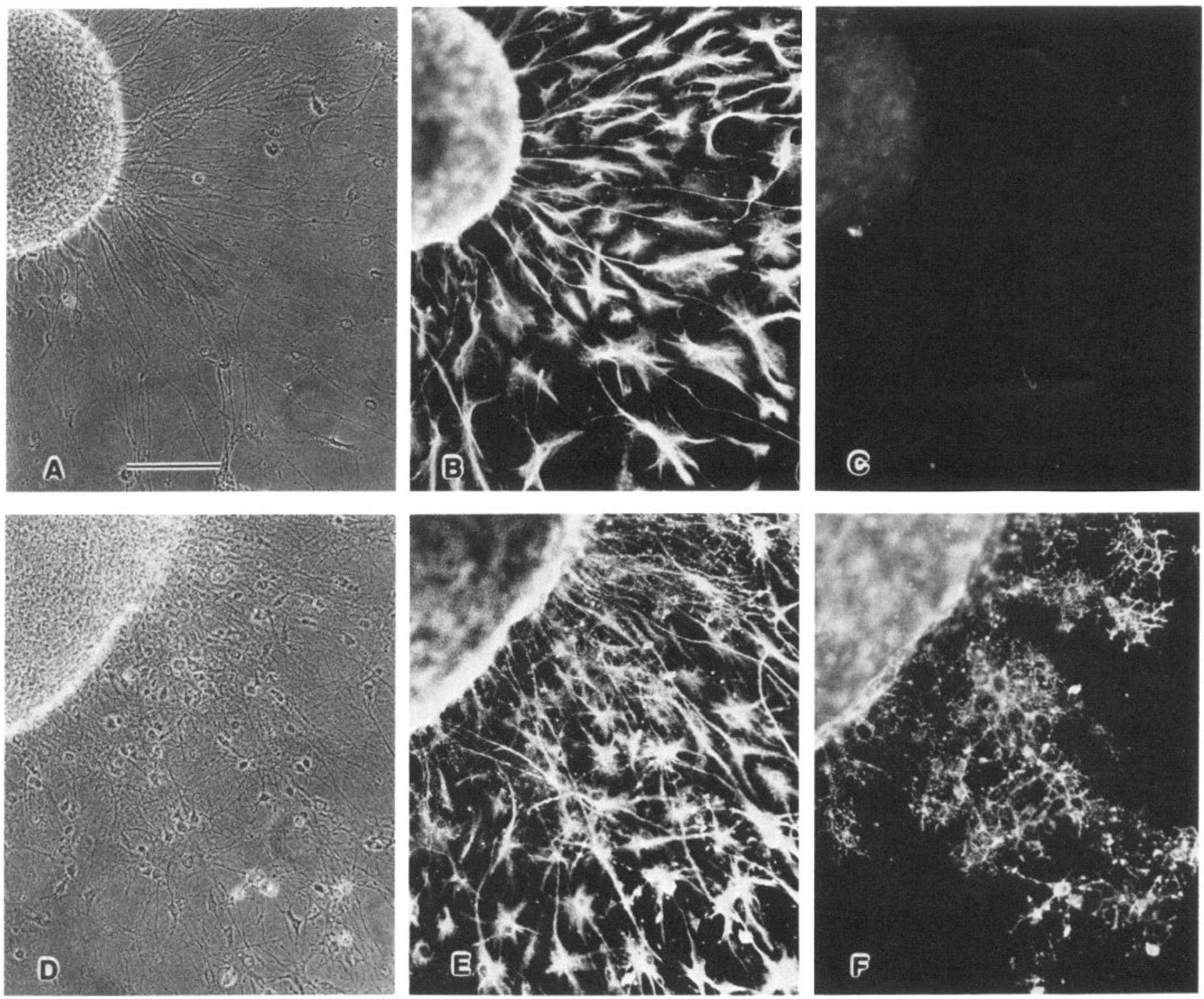

Figure 2. Explant cultures derived from either dorsal $(A-C)$ or ventral $(D-F)$ E14 thoracolumbar spinal cord after $9 \mathrm{~d}$ in culture. Cultures were examined with phase optics $(A$ and $D)$ and following double immunofluorescent labeling with anti-GFAP antibodies $(B$ and $E)$ and anti-GC antibodies $(C$ and $F)$. While explants from both dorsal and ventral spinal cord yielded large numbers of GFAP+ astrocytes $(B$ and $E)$, GC+ oligodendrocytes developed only from the ventrally derived explants $(C$ and $F)$. Scale bar, $100 \mu \mathrm{m}$.

is that oligodendrocytes or their precursors arise in ventral spinal cord and then migrate dorsally during development. To determine if ventral-to-dorsal migration occurred in embryonic spinal cord, cells in the ventral region of E18 spinal cord segments were locally labeled with DiI (Fig. 4). In approximately $50 \%$ of the live segments, DiI-labeled cells were located in the presumptive dorsal funiculi $18 \mathrm{hr}$ later (Fig. 4). Isolated labeled cells were also seen in the dorsolateral and lateral funiculi. In control experiments, when the distribution of label was examined immediately after DiI labeling, or following initial fixation and then $18 \mathrm{hr}$ of incubation, the label was localized only at the ventral region of the spinal cord and no labeled cells were seen in the dorsal funiculi of any segments (Fig. 4). These observations suggest that the labeled cells had migrated from ventral to dorsal regions in the living spinal cord segments.

To determine whether the cells that migrated from ventral to dorsal locations had the capacity to develop into oligodendro- cytes, DiI labeling experiments were repeated on E21 spinal cords followed by labeling anti-GC antibodies. E21 spinal cords were chosen for two reasons. First, many more GC+ cells are present in the dorsal spinal at E21 than at earlier ages, and, second, the larger size of the axial segment allowed for accurate ventral application of the DiI crystal. As in the experiments on E18 spinal cords, $18 \mathrm{hr}$ after DiI labeling of the ventral quarter of the cord, DiI-labeled cells were detected in the dorsal funiculi of the E21 spinal cord. Double-labeling with anti-GC antibodies revealed that some of the DiI-labeled cells in the dorsal funiculi were GC+ oligodendrocytes (Fig. 5). The majority of dorsally located GC+ oligodendrocytes were not labeled with $\mathrm{DiI}$ in these experiments. However, since dissociated culture experiments suggested that considerable migration of oligodendrocyte precursors had occurred as early as E18 (Table 2), it seems probable that the GC+ DiI-labeled cells seen at E21 developed from cells that had migrated from ventral regions before E21. 

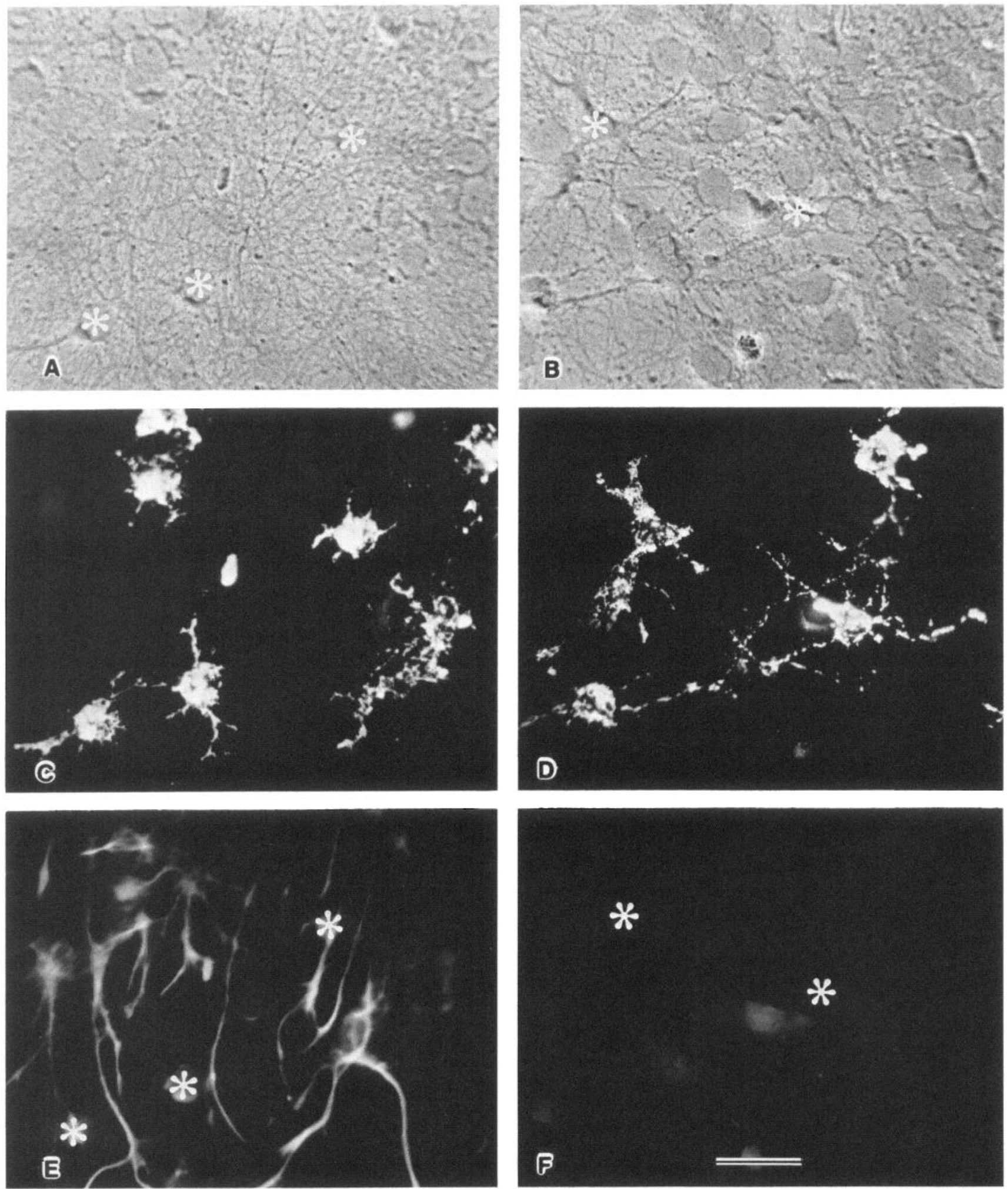

Figure 3. The majority of cells that migrate from an E14 ventrally derived spinal cord explant have the antigenic characteristics of progenitor cells and not oligodendrocytes. $A$ and $B$, Phase contrast; $C$ and $D$, A2B5 labeling visualized with rhodamine optics; $D$, anti-GFAP labeling of the same field as in panels $A$ and $C$, visualized with fluorescein optics; $E$, anti-GC labeling of the same field as in panels $B$ and $D$, visualized with fluorescein optics. Note that the migratory cells (asterisks) are process-bearing and have A2B5 immunoreactivity but not anti-GFAP or anti-GC immunoreactivity. Scale bar, $30 \mu \mathrm{m}$. 

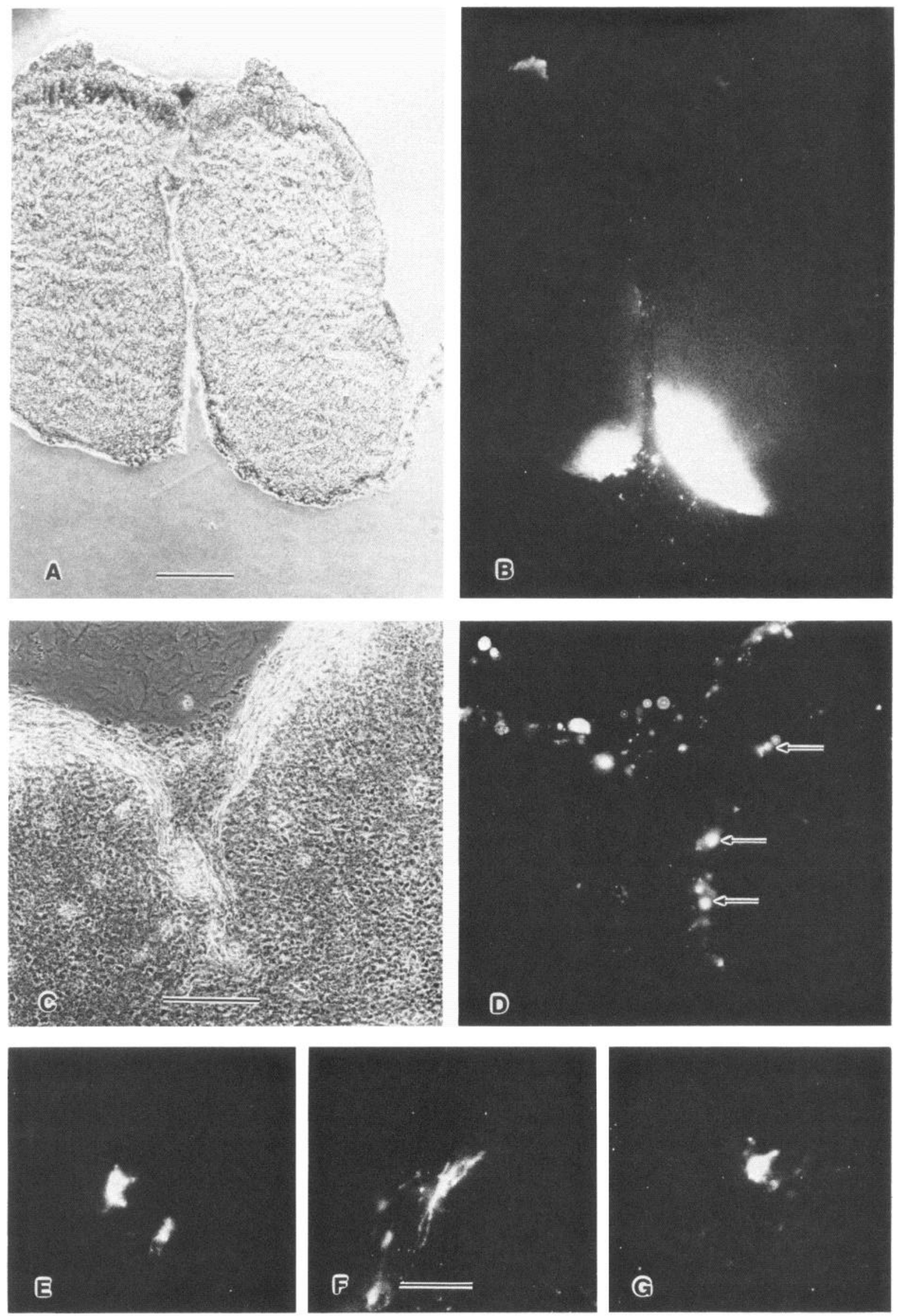

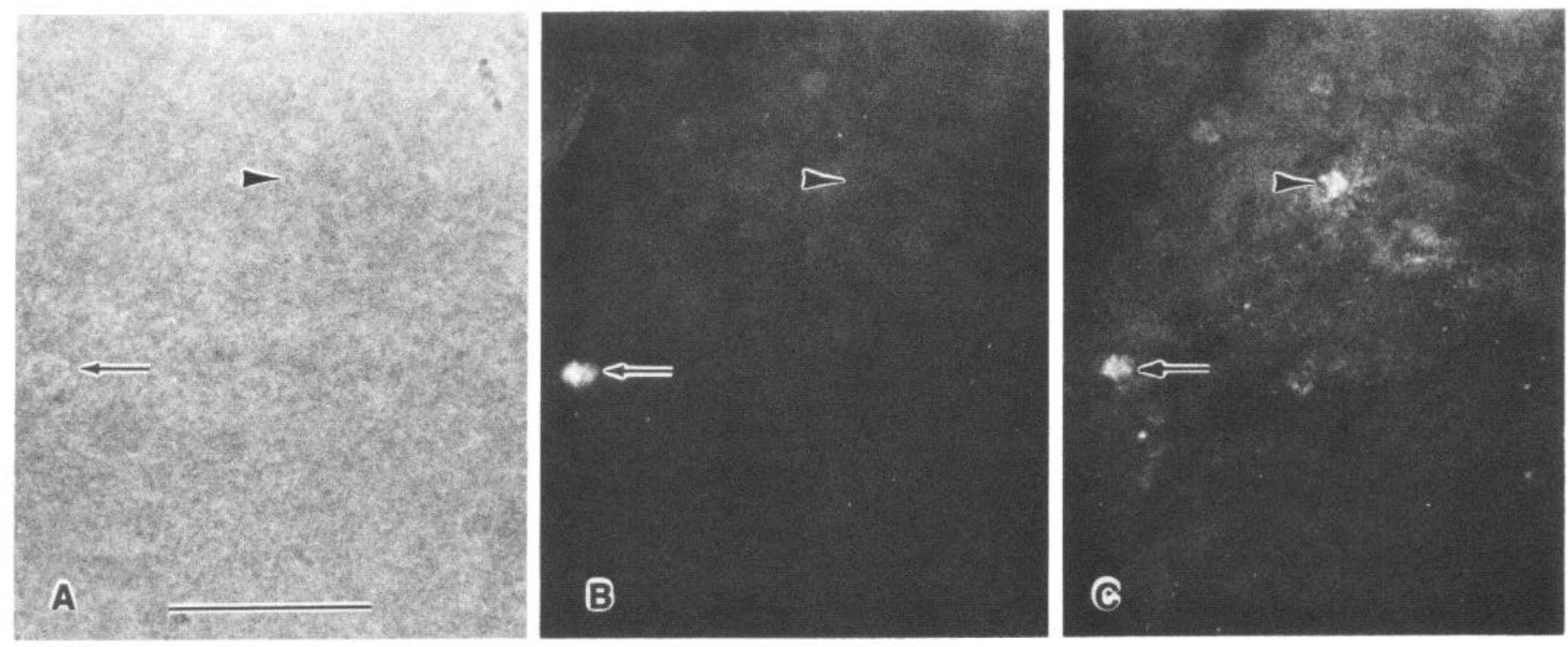

Figure 5. The dorsal region of an E21 sagittal segment of rat spinal cord, $18 \mathrm{hr}$ after application of DiI label to the ventromedial surface. A, Phase contrast; $B$, DiI labeling visualized under rhodamine optics; $C$, anti-GC labeling visualized under fluorescein optics. The ventrally derived DiIlabeled cell (arrow in panels $B$ and $C$ ) has begun to express GC (arrow in panel $C$ ). Another GC-positive cell (arrowhead) is not DiI-labeled, possibly because it had left the ventral region of the cord before the application of DiI. Scale bar, $100 \mu \mathrm{m}$.

That not all the dorsally located DiI-labeled cells stained with anti-GC antibodies may reflect the migration of precursor cells rather than GC+ oligodendrocytes. The observation that cells in the dorsal funiculi at E21 expressed both DiI and GC provides strong evidence that at least some oligodendrocytes in the dorsal funiculi developed from cells originally located ventrally in the spinal cord.

\section{Discussion}

In this study, we show that the differentiation of oligodendrocytes in the rat spinal cord occurs in a rostrocaudal and ventrodorsal sequence. At E14, all segmental levels of the spinal cord have the capacity for oligodendrogenesis. However, in thoracolumbar segments at E14, the ability to give rise to oligodendrocytes is restricted to ventral regions of the spinal cord. During subsequent development, dorsal regions aquire the capacity for oligodendrogenesis through the ventral-to-dorsal migration of oligodendrocyte precursors.

While the role of oligodendrocytes in myelin formation in the spinal cord (Meinecke and Webster, 1984) and other regions of the CNS is clear (Bunge, 1968; Peters et al., 1976), the origin of oligodendrocytes in the spinal cord is not well understood. A number of explanations may account for the appearance of oligodendrocytes in the developing spinal cord. For example, oligodendrocytes may be generated at specific rostral-caudal levels of the CNS and migrate longitudinally through the spinal cord during development. Alternatively, oligodendrocytes may be generated from cells around the central canal at all levels of the spinal cord and migrate radially to the white matter (Gilmore, 1971; Sturrock, 1982). Finally, oligodendrocytes may be derived directly from the radial glial cells of the embryonic spinal cord (Choi et al., 1983; Choi and Kim, 1985; Hirano and Goldman, 1988). To distinguish between these possibilities, the development of oligodendrocytes and the capacity of different regions of the spinal cord to give rise to oligodendrocytes in vitro were examined. Oligodendrocytes were found to develop in both a rostrocaudal and ventrodorsal sequence, consistent with previous studies that have suggested a similar developmental sequence for neuronal and glial cell populations during spinal cord development (Nornes and Das, 1974; Sturrock, 1982).

The timing of spinal cord oligodendrocyte differentiation does not require the presence of an intact spinal cord. In cultures established from either E14 or E16 thoracolumbar spinal cord, the first GC+ oligodendrocytes appeared at the equivalent age of E17 (that is, after 3 or $1 \mathrm{~d}$ in culture, respectively). This is the same age at which GC+ oligodendrocytes were first detected in freshly isolated cell suspensions. It seems that the capacity for oligodendrocytes to develop on schedule in culture is a general phenomenon, as GC+ oligodendrocytes appear at approximately the same equivalent age in cultures of embryonic brain as they do in vivo (Abney et al., 1981; Williams et al., 1985).

If oligodendrocyte precursors migrated longitudinally down the spinal cord from a localized source, this migration must have occurred prior to E14. When isolated cervical, thoracic,

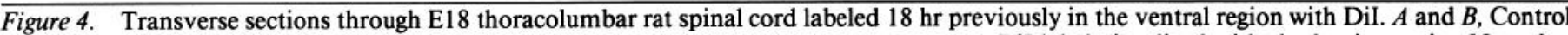

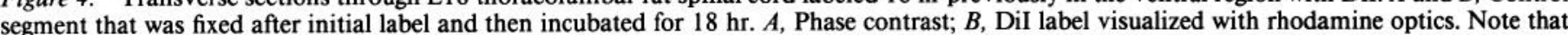

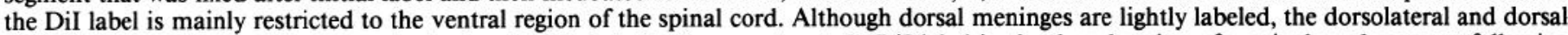

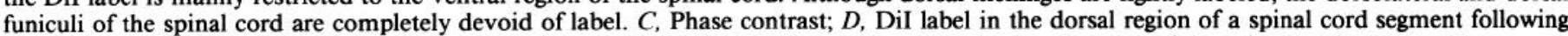

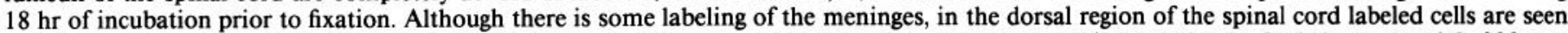

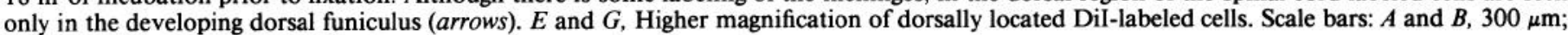
$C$ and $D, 100 \mu \mathrm{m} ; E$ and $G, 40 \mu \mathrm{m}$. 
or lumbar segments of E14 spinal cord, including both dorsal and ventral regions, were grown for $9 \mathrm{~d}$ in vitro, oligodendrocytes developed in all cultures. These observations indicate that all segments of the spinal cord contained oligodendrocyte precursors by that age. While early longitudinal migration of oligodendrocyte precursors cannot be ruled out, it seems unlikely given the general immaturity of the spinal cord at E14. A more likely explanation is that oligodendrocyte precursors arise from neuroepithelial cells at all levels of the spinal cord.

Although oligodendrocytes developed in cultures derived from all segmental levels of the spinal cord at E14, the potential for oligodendrogenesis appeared to be restricted to ventral regions of the thoracolumbar cord at that age. Large numbers of oligodendrocytes developed after $9 \mathrm{~d}$ in all cultures derived from ventral spinal cord, whilc virtually no oligodendrocytes developed in culture derived from dorsal spinal cord of the same animals; this was the case even if the cultures were allowed to develop for a further $10 \mathrm{~d}$. The absence of oligodendrocytes in E14 cultures from dorsal cord is unlikely to be the result of selective death or failure of proliferation of precursor cells in these cultures, since numerous oligodendrocytes developed in dorsal cultures established from E1 8 spinal cord. Furthermore, the addition of variable numbers of dorsally derived cells to cultures containing a constant number of ventrally derived cells did not affect the number of oligodendrocytes that developed. Furthermore, in explant cultures that preserve the local cytoarchitecture, oligodendrocytes developed in the majority of ventral but less than $1 \%$ of the dorsal cultures. It is unlikely that oligodendrocyte differentiation was dependent on local cell-cell interactions that were disrupted by cell dissociation.

The most likcly cxplanation for the lack of oligodendrogenesis in isolated E14 dorsal spinal cord is that oligodendrocyte precursor cells are not present in the dorsal thoracolumbar spinal cord at that age. According to this proposal, oligodendrocyte precursors that were initially located ventrally migrate dorsally and differentiate into oligodendrocytes. A number of observations are consistent with this hypothesis. First, the majority of $\mathrm{GC}+$ cells that developed from E14 ventral explants were located some distance from the bulk of the explant, demonstrating that spinal cord oligodendrocyte precursors have the capacity to migrate. Second, cells labeled by the application of DiI to the ventral spinal cord were found in the developing dorsal funculi in segments of E18 spinal cord, showing that ventrodorsal cell migration occurred; and, third, in E21 spinal cord segments, some of the dorsally located DiI-labeled cells were also $\mathrm{GC}+$, showing that the migratory cells gave rise to oligodendrocytes.

The characteristics of the ventrodorsal migrating oligodendrocyte precursor cells in spinal cord are unknown. These cells may be immature, but committed, oligodendrocytes. The capacity of immature oligodendrocytes or their precursors to $\mathrm{mi}$ grate long distances through the CNS has previously been shown using transplantation techniques (La Chapelle et al., 1984). Alternatively, the ventrodorsal migrating cells may be progenitor cells whose fate is restricted but that are not committed to the oligodendrocyte pathway. The best known example of restricted progenitor cells is the $\mathrm{O}-2 \mathrm{~A}$ progenitor cell initially described in cultures from the rat optic nerve (Raff et al., 1983b) and more recently in other regions of the CNS (Levi et al., 1986b; Behar et al., 1988). In culture, O2-A progenitor cells show A2B5 immunoreactivity and are bipotential, differentiating into either oligodendrocytes or type- 2 astrocytes depending on environ- mental signals (Raff, 1989). In vitro, progenitor cells are highly motile until they differentiate into oligodendrocytes (Temple and Raff, 1986; Small et al., 1987), and indirect evidence suggests that $\mathrm{O}-2 \mathrm{~A}$ progenitor cells migrate into the optic nerve from the brain during development (Small et al., 1987).

It seems likely that the ventrodorsal migrating oligodendrocyte precursors in the embryonic rat spinal cord are analogous to O-2A progenitor cells for a number of reasons. First, at the time when this migration commences (E15-16), there are no cells in the spinal cord that possess GC immunoreactivity, a marker of mature oligodendrocytes (Raff et al., 1978). Second, many of the cells in dorsal funiculi that had been labeled by the ventral application of DiI at E21 lacked GC immunoreactivity. Finally, many of the cells which initially migrated from the explants of E1 4 ventral spinal cord had a process-bearing morphology similar to O-2A progenitor cells and labeled with A2B5 but not with antibodies against GC or GFAP. Preliminary studies suggest that at least some of these migrating cells can give rise to either oligodendrocytes or type-2-like astrocytes in vitro depending on the culture environment (J. Fok-Seang and R. H. Miller, manuscript in preparation).

Although at E14 dorsal spinal cord appeared to lack the capacity to give rise to oligodendrocytes, it did have the capacity to give rise to astrocytes and contained vimentin immunoreactive radial glial cells (B. C. Warf, J. Fok-Seang, and R. H. Miller, unpublished observation). It therefore seems likely that the radial glial cells in the dorsal region of the thoracolumbar spinal cord are not the immediate precursors of oligodendrocytes. If oligodendrocytes did develop directly from radial glia as proposed (Choi and Kim, 1983; Choi et al., 1985; Hirano and Goldman, 1988), this transformation may occur only from the radial glial cells of the ventral spinal cord.

It is unclear why astrocytes but not oligodendrocytes develop from the glial precursors in the early embryonic dorsal spinal cord. It may be that glial precursors in the dorsal region lack the capacity to produce oligodendrocytes or that they fail to receive some inductive signal. If such an inductive signal exists, the source must be located ventrally. The absence of dorsally derived oligodendrocytes in dorsal-ventral coculture experiments made it unlikely that such a signal is transmitted by ventral spinal cord cells themselves. Furthermore, the absence of dorsally derived oligodendrocytes after coculture with notochord and other ventral tissue masses suggest that even if such a ventral signal were present, dorsal spinal cord cells may be unresponsive.

One attractive hypothesis to explain the initial ventral location of oligodendrocyte precursors is that a source of the oligodendrocyte inductive signal that may commit glial precursors to the O-2A lineage is located outside the CNS, possibly in the notochord, and that only ventrally located spinal cord cells have the capacity to respond. Indeed, in the chick, transplantation of the notochord has been shown to have a profound effect on spinal cord development in the region of the transplanted notochord (Van Stratten et al., 1989), and more recently both the ventral floor plate region of the spinal cord and the notochord have been shown to be a source of morphogens including retinoic acid (Wagner et al., 1990). Since retinoic acid influences both neuronal and astroglial spinal cord cell differentiation in vitro (Wuarin et al., 1990), it is possible that the localized concentration of retinoic acid in the ventral region of the spinal cord may also influence the differentiation of spinal cord oligodendrocytes. 


\section{References}

Abney, ER, Bartlett PF, Raff MC (1981) Astrocytes, ependymal cells and oligodendrocytes develop on schedule in dissociated cell cultures of embryonic rat brain. Dev Biol 83:301-310.

Altman J, Bayer SA (1984) The development of the rat spinal cord. Adv Anat Embryol Cell Biol 85:000-000.

Bartlett PF, Noble M, Pruss RM, Raff MC, Rattray S, Williams CA (1981) Rat neural antigen-2 (Ran-2): a cell surface antigen on astrocytes, ependymal cells, Muller cells and leptomeninges defined by a monoclonal antibody. Brain Res 204:339-351.

Behar T, McMorris FA, Novotny EA, Barker JL, Dubois-Dalacq M (1988) Growth and differentiation properties of O-2A progenitors purified from rat cerebral hemispheres. J Neurosci Res 21:168-180.

Bignami A, Dahl D (1974) Astrocyte specific protein and radial glia in the cerebral cortex of new born rat. Nature 252:55-56.

Bignami A, Eng LF, Dahl D, Uyeda CT (1972) Localization of the glial fibrillary acidic protein in astrocytes by immunofluorescence. Brain Res 43:429-435.

Bottenstein JE, Sato GH (1979) Growth of a rat neuroblastoma cell line in serum free supplemented medium. Proc Natl Acad Sci USA $76: 514-517$.

Brown AG (1981) Organization in the spinal cord. Berlin: Springer.

Bunge RP (1968) Glial cells and the central myelin sheath. Physiol Rev 48:197-251.

Choi BH, Kim RC (1985) Expression of glial fibrillary acidic protein by immature oligodendroglia and its implications. J Neuroimmunol 8:215-235.

Choi BH, Kim RC, Lapham LW (1983) Do radial glia give rise to both astroglial and oligodendroglial cells? Dev Brain Res 8:119-130.

Culican SM, Baumrind NL, Yamamoto M, Pearlman AL (1990) Cortical radial glia: identification in tissue culture and evidence for their transformation to astrocytes. J Neurosci 10:684-692.

Eisenbarth GS, Walsh FS, Nirenberg M (1979) Monoclonal antibody to a plasma membrane antigen of neurons. Proc Natl Acad Sci USA $76: 4913-4917$

Fujita S (1965) An autoradiographic study on the origin and fate of the sub-pial glioblast in the embryonic chick spinal cord. J Comp Neurol 124:51-50.

Gilmore SA (1971) Neuroglial population in the spinal white matter of neonatal and early postnatal rats: an autoradiographic study of numbers of neuroglia and changes in their proliferative activity. Anat Rec 171:283-292.

Godement P, Vanselow J, Thanos S, Bonhoeffer F (1987) A study in developing visual systems with a new method of staining neurones and their processes in fixed tissue. Development 101:697-713.

Hatten ME (1990) Riding the glial monorail: a common mechanism for glial-guided neuronal migration in different regions of the development mammalian brain. Trends Neurosci 13:179-184.

Hertz L (1981) Functional interactions between astrocytes and neurons. In: Glial and neuronal cell biology, (Fedoroff S, ed), pp 45-58. New York: Liss.

Hirano M, Goldman JE (1988) Gliogenesis in rat spinal cord: evidence for origin of astrocytes and oligodendrocytes from radial precursors. J Neurosci Res 21:155-167.

Honig MG, Hume RI (1986) Fluorescent carbocyanine dyes allow living neurons of identified origin to be studied in long-term culture. J Cell Biol 103:171-187.

Hughes SM, Raff MC (1987) An inducer protein may control the timing of fate switching in a bipotential glial progenitor cell in the rat optic nerve. Development 101:157-167.

Janzer RC, Raff MC (1987) Astrocytes induce blood brain barrier properties in endothelial cells. Nature 325:253-257.

Kingsbury BF (1926) On the so-called law of anteroposterior development. Anat Rec 33:73-87.

La Chapelle F, Gumpel M, Baulac M, Jacque C, Due P, Baumann N (1984) Transplantion of CNS fragments into the brain of shiverer mutant mice: extensive myelination by implanted oligodendrocytes. I. Immunological studies. Dev Neurosci 6:325-334.

Levi G, Gallo V, Wilkins GP, Cohen J (1986a) Astrocyte subpopulations and glial precursors in rat cerebellar cell cultures. Adv Biosci $61: 21-30$.

Levi G, Gallo V, Ciotti MT (1986b) Bipotential precursors of putative fibrous astrocytes and oligodendrocytes in rat cerebellar cultures ex- press distinct surface features and "neuron-like"-aminobutyric acid transport. Proc Natl Acad Sci USA 83:1504-1508.

Levitt P, Rakic P (1980) Immunoperoxidase localization of glial fibrillary acidic protein in radial glial cells and astrocytes of the developing rhesus monkey brain. J Comp Neurol 193:815-840.

Lillien LE, Raff MC (1990) Analysis of the cell-cell interactions that control type-2 astrocyte development in vitro. Neuron 4:525-534.

Ling EA (1976) Study in the changes of the proportions and numbers of the various glial cell types in the spinal cord of neonatal and young adult rats. Acta Anat 96:188-195.

Meinecke DL, de F Webster H (1984) Fine structure of dividing astroglia and oligodendroglia during myelin formation in the developing mouse spinal cord. J Comp Neurol 222:47-55.

Noble M, Murray K, Stroobant P, Waterfield MD, Riddle P (1988) Platelet-derived growth factor promotes division and motility and inhibits premature differentiation of the oligodendrocyte/type- 2 astrocyte progenitor cell. Nature 333:560-562.

Nornes HO, Das GD (1974) Temporal pattern of neurogenesis in spinal cord of Rat. I. An autoradiographic study-time and sites of origin and migration and settling patterns of ncuroblasts. Brain Res 73:121-138.

Peters A, Palay SL, de F Webster H (1976) The fine structure of the nervous system: the neurones and the supporting cells. Philadelphia: W. B. Saunders.

Privat A, Leblond CP (1972) The subependymal layer and neighbouring region in the brain of the young rat. J Comp Neurol 146: 277-302.

Pruss, R (1979) Thy-1 antigen on astrocytes in long term cultures of rat central nervous system. Nature 280:688-690.

Raff MC (1989) Glial cell diversification in the optic nerve. Science 243:1450-1455.

Raff MC, Mirsky R, Fields KL, Lisak RP, Dorfman SH, Silberberg DH, Gregson NA, Liebowitz S, Kennedy MC (1978) Galactocerebroside is a specific cell surface antigenic marker for oligodendrocytes in culture. Nature 274:813--816.

Raff MC, Abney ER, Cohen J, Lindsay R, Noble M (1983a) Two types of astrocytes in cultures of developing rat white matter: differences in morphology, surface gangliosides, and growth characteristics. J Neurosci 3:1289-1300.

Raff MC, Miller RH, Noble M (1983b) A glial progenitor cell that develops in vitro into an astrocyte or an oligodendrocyte depending on the culture medium. Nature 303:390-396.

Raff MC, Abney ER, Miller RH (1984) Two glial cell lineages diverge prenatally in the rat optic nerve. Dev Biol 106:53-60.

Rakic P (1971) Neuron-glial relationship during granule cell migration in developing cerebellar cortex. A Golgi and electronmicroscopic study in Maccacus rhesus. J Comp Neurol 141:238-312.

Ramon y Cajal S (1909) Histologie du système nerveux de l'homme et des vertebres, pp 230-252. Paris: Maloine (reprinted, Madrid: Consejo Superior de Investigaciones Cientificas, 1952).

Ranscht B, Clapshaw PA, Price J, Noble M, Seifert W (1982) Development of oligodendrocytes and Schwann cells studied with a monoclonal antibody against galactocerebroside. Proc Natl Acad Sci USA 79:2709-2713

Retzius G (1898) Zur Frage vo der Endigungweise der peripherischen sensiblen Nerven. Biol Untersuch 8:114-128

Rexed B (1952) The cytoarchitectonic organization of the spinal cord in the cat. J Comp Neurol 96:415-495.

Richardson WD, Pringle N, Mosley MJ, Westermark B, Dubois-Galcq M (1988) A role for platelet-derived growth factor in normal gliogenesis in the central nervous system. Cell 53:309-319.

Schmechel DE, Rakic P (1979) A Golgi study of radial glial cells in developing monkey telencephalon: morphogenesis and transformation into astrocytes. Anat Embryol 156:115-152.

Silver J, Lorenz SE, Wahlsten D, Coughlin J (1982) Axonal guidance during the development of the great cerebral commissures: descriptive and experimental studies in vivo on the role of preformed glial pathways. J Comp Neurol 210:10-29.

Small RK, Riddle P, Noble M (1987) Evidence for migration of oligodendrocyte-type-2 astrocyte progenitor cells into the developing rat optic nerve. Nature 328:155-157.

Smart I (1961) The subependymal layer of the mouse brain and its cell production as shown by radioautography after thymidine- $\mathrm{H} 3$ injection. J Comp Neurol 116:325-347.

Smith GM, Rutishauser U, Silver J, Miller RH (1990) Maturation of 
astrocytes in vitro alters the extent and molecular basis of neurite outgrowth. Dev Biol 138:377-390.

Sturrock RR (1982) Gliogenesis in the prenatal rabbit spinal cord. J Anat 134:771-793.

Temple S, Raff MC (1986) Clonal analysis of oligodendrocyte development in culture: evidence for a developmental clock that counts cell divisions. Cell 44:773-779.

Van Stratten HWM, Hekking JWM, Beursgen JPWM, Terwindt-Rouwenhorst E, Drukker J (1989) Effect of the notochord on proliferation and differentiation in the neural tube of the chick embryo. Development 107:793-803
Wagner M, Thaller C, Jessel T, Eichele G (1990) Polarizing activity and retinoid synthesis in the floor plate of the neural tube. Nature 345:819-822

Williams BP, Abney ER, Raff MC (1985) Macroglial cell development in embryonic rat brain: studies using monoclonal antibodies, fluorescence activated cell sorting and cell culture. Dev Biol 112:126134.

Wuarin L, Sidell N, de Vellis J (1990) Retinoids increase perinatal spinal cord neuronal survival and astroglial differentiation. Int $J$ Dev Neurosci 8:317-326. 